\title{
IV Gesetzgeberische Relativierung der namensrechtlichen Individualisierungs- und Klassifizierungsfunktion aus übergeordneten Gründen
}

Der deutsche Gesetzgeber hat im Zuge der großen familienrechtlichen Reformen in der zweiten Hälfte des 20. Jahrhunderts zunehmend die Individualisierungsund Klassifizierungsfunktion des Namens relativiert, und zwar vornehmlich nicht aus namensrechtsspezifischen, sondern aus übergeordneten Gründen.

\section{Vorab: Name als taugliches und legitimes Individualisierungs- und Klassifizierungsmittel nicht in Frage gestellt}

Diese Entwicklung war weniger den bereits erwähnten (oben III. 1.) Schwächen des Namens als Mittel zur Individualisierung und Klassifizierung und den Spannungen zwischen Individualisierungs- und Klassifizierungsfunktion geschuldet.

Auch Zweifel an der Legitimität einer Klassifizierungsfunktion des Namens haben den Reformgesetzgeber offenbar nicht geleitet - überraschenderweise. Denn es ist nicht ersichtlich, welches Interesse ein Gesetzgeber daran haben kann, dass der - meist auch gesellschaftlich verwendete (vgl. aber unten VI.) und damit potentiell für jedermann sichtbare - rechtliche Name bestimmte Charakteristika der Person offenlegt. ${ }^{49}$ Im Gegenteil: Die von den Nationalsozialisten im Jahr 1938 mit der Zweiten Verordnung zur Durchführung des Gesetzes über die Änderung von Familiennamen und Vornamen ${ }^{50}$ eingeführten jüdischen Zwangsvornamen „Israel“ und „Sara“ für jüdische Bürger (§ 2 Abs. 1 der Verordnung) belegen, zu welchen Perversionen die Klassifizierungsfunktion des Namens genutzt werden kann. ${ }^{51}$ Den rechtlichen Namen setzten die Nationalsozialisten übrigens auch in

49 Anders aber offenbar Albrecht Dieckmann Zur Namensführung des Kindes, StAZ 1982, 266 (278), der die „Ordnungskraft“ des Namens „im öffentlichen Bereich“ hervorhebt und zu bedenken gibt, dass Kennziffern „nur für ,Eingeweihte“ - nicht für die Allgemeinheit“ verständlich seien.

50 Vom 17.8.1938, RGBl. 1938 I S. 1044.

51 Zum Zusammenhang zwischen behördlicher Namensänderung in Deutschland und Judenemanzipation einerseits bzw. Antisemitismus andererseits siehe die Studie von Michael WagnerKern Staat und Namensänderung (2002).

Ә OpenAccess. (๑) 2020 Juristische Gesellschaft zu Berlin, publiziert von De Gruyter.

(c) BY-NC-ND Dieses Werk ist lizenziert unter der Creative Commons Attribution-NonCommercial-NoDerivatives 4.0 License. https://doi.org/10.1515/9783110709872-005 
anderem Zusammenhang als Klassifizierungsmittel ein: Die Lex Krupp des Jahres 1943 gestattete der Familie Krupp nicht nur, ein Sondererbrecht für die Nachfolge in das Kruppsche Familienvermögen zu schaffen, ${ }^{52}$ sondern sah auch vor, dass der jeweilige Erbe den Namen „Krupp“ vor seinem Familiennamen führt ${ }^{53}$ und damit nach außen sichtbar als Krupperbe gekennzeichnet war. Das Interesse an der Bewahrung einer bestimmten Namenskultur in einer Gesellschaft, die den Namen traditionell als Klassifizierungsmittel nutzt, ist kein sinnvolles gesetzgeberisches Anliegen, zumindestens in einem freiheitlichen Staat, der seinen Bürgern kein kulturell bedingtes Verhalten aufoktroyieren möchte. Dennoch wurde die Klassifizierungsfunktion des Namens vor allem in Deutschland lange vehement verteidigt, speziell diejenige des Familiennamens im Hinblick auf eine Familienzugehörigkeit. $^{54}$

52 Erlass des Führers über das Familienunternehmen der Firma Fried. Krupp vom 12.11.1943, RGBl. 1943 I S. 655, Ziff. I.

53 Ziff. III des Erlasses.

54 Nachdrücklich etwa noch Nelle (Fn. 32) 811 f.: „Die Frage, ob vor diesem Hintergrund die Aufrechterhaltung einer familialen Zuordnungsfunktion überhaupt noch gerechtfertigt ist, hat daher längst schon keinen provokativen Charakter mehr. Bei der Entscheidung dieser Frage ist zunächst zu berücksichtigen, daß im täglichen Leben ein unabweisbares Bedürfnis danach besteht, eine Familie unter einem Sammelnamen anzureden [...]. Auch ist ein gemeinschaftlicher Familienname als Kristallisationspunkt für die Entwicklung und Pflege eines Zusammengehörigkeitsgefühls der sozialen Einheit Familie keineswegs unwesentlich [...]. Würde man der Familie die Identifikationsmöglichkeit des gemeinschaftlichen Namens versagen, stünde sie sogar schlechter als bloße Zweckgemeinschaften wie der Verein oder die Gesellschaft [...]. Auch aus der Sicht der Allgemeinheit gibt es gute Gründe für eine Verlautbarung von Familienzugehörigkeiten, man denke etwa an die Mithaftung für Geschäfte des Ehepartners oder die Aufsichtspflicht für die Kinder [...]. Ebenso erscheint es wünschenswert, daß eine Verwaltung, die familienfreundlich handeln soll, die Familienzugehörigkeit schon am Namen erkennen kann“. Allerdings möchte Nelle aaO, die Klassifizierungsfunktion des Namens auf die Familienzugehörigkeit beschränken: „Über die Familienzugehörigkeit hinaus lässt der Name noch ein weitgespanntes Geflecht sozialer Verbindungen anklingen, wie etwa die bereits erwähnte Zugehörigkeit zu einem Adelsgeschlecht, oder aber auch eine bestimmte geographische oder ethnische Herkunft [...]“ Allerdings handele „es sich bei den aufgezählten Aspekten um keine Gesichtspunkte, an deren Verlautbarung ein schützenswertes Interesse Dritter ersichtlich wäre oder welche ein zulässiges Kriterium für die Entscheidung von Verwaltungsvorgängen bilden könnten, vielmehr würde umgekehrt geradezu ein Ansatzpunkt für unzulässige Diskriminierungen geschaffen. [...] Eine über die Familienzugehörigkeit hinausgehende Zuordnungsfunktion des Namens ist somit abzulehnen“. Noch einem gänzlich anderen Familien- und Gesellschaftsmodell (und offenbar auch Verfassungsmodell) verhaftet und unter Rückgriff auf die „überkommene[...] Volksordnung“ und die „Anschauungen unseres Volkes“ Graf von Bernstorff Der Familienname in der deutschen Rechtsordnung, NJW 1957, 1901: „Eine Verwirklichung des Vorschlags der freien Namenswahl durch die Ehegatten hätte dem Familiennamen seine entscheidende Funktion genommen, im Sinne der Gliederung des Volkes in Sippen und Familien alle die zu kennzeichnen, die im Mannesstamm aus der gleichen 
Das heute anzutreffende Normendickicht ist also weniger der Auseinandersetzung mit den klassischen Namensfunktionen geschuldet. Vielmehr war ein bedingungsloses Festhalten an der Individualisierungs- und Klassifizierungsfunktion aus übergeordneten Gründen nicht mehr möglich, die teils auch verfassungsrechtlichen oder unionsrechtlichen Ursprungs sind. ${ }^{55}$ Im Folgenden möchte ich vier Gründe für die Relativierung der traditionellen Namensfunktionen anreißen.

\section{Gleichheit vor dem Gesetz}

Es zeigte sich bereits rasch nach Inkrafttreten des Grundgesetzes, dass eine namensrechtliche Klassifizierung nach der Familienzugehörigkeit des Namensträgers vor dem Hintergrund des Gleichheitssatzes nicht unproblematisch ist.

\section{a) Klassifizierungsfunktion im Hinblick auf die Zugehörigkeit des Namensträgers zu einer bestimmten Familiendynastie per se gleichheitswidrig}

Das dynastische Denken im ursprünglichen Namensrecht (oben III. 1.) war kaum mit der Gleichberechtigung von Mann und Frau (Art. 3 Abs. 2, 3 GG) vereinbar. Widerstand regte sich bereits früh. Sieben Jahre nach dem Anwendungsbeginn des Bürgerlichen Gesetzbuchs sprach Marianne Weber - die unter der Knute des $\S 1355$ BGB a.F. (1900) den Namen ihres Mannes Max Weber als Ehenamen tragen musste - in ihrer Studie zur „Ehefrau und Mutter in der Rechtsentwicklung“ dem damaligen Ehenamensrecht jeglichen „normativen Charakter“ ab: Die Vorschrift sei ein „Rest primitiven, auf Grund des Frauenkaufs erworbenen ,Vaterrechts“, unter dem die gesetzlich noch nicht als Persönlichkeit gewertete Frau gar keine

\footnotetext{
Familie stammen. Mit solcher Funktion enthält der Familienname eine über die rechtlichen Beziehungen zweier Familienmitglieder weit hinausgreifende Bedeutung, indem er in die Vergangenheit hinein seinen Träger mit seinen Vätern und Vorvätern und mit deren geistiger Welt und Leistung verbindet [...] und schließlich auch seinen Träger zum Vermittler des geistigen und sittlichen Erbguts der Familie gegenüber Kindern und Kindeskindern macht“.

55 Hierauf weist bereits Thomas Wagenitz Grundlinien des neuen Familiennamensrechts, FamRZ 1994, 409 (416) hin, der beklagt, dass das Ringen „um das richtige Ehe- oder Familienbild, um recht verstandene Gleichberechtigung, um Menschenwürde“ die Arbeit des Gesetzgebers im Namensrecht besonders schwer mache.
} 
rechtliche Sonderexistenz besaß, sondern ihren Namen und Stand so gut wie ihr Recht vom Manne empfing. “56

Allerdings ermöglichte erst das Gleichberechtigungsgesetz des Jahres $1957^{57}$ der Ehefrau, dem Geburtsnamen des Mannes, der weiterhin Ehename wurde, ihren eigenen Geburtsnamen - das Gesetz sprach noch vom „Mädchennamen“ anzufügen (§ 1355 S. 2 Halbsatz 1 BGB a.F. [1957]). Erst das für das Familienrecht epochale Erste Eherechtsreformgesetz, ${ }^{58}$ dessen Inkrafttreten sich vor zwei Jahren zum vierzigsten Mal jährte, ${ }^{59}$ gestattete den Ehegatten, zwischen dem Geburtsnamen des Mannes und demjenigen der Frau zu wählen (§ 1355 Abs. 2 S. 1 BGB a.F. [1976]). Allerdings kam der Mannesname zum Zuge, wenn sich die Ehegatten nicht einigen konnten (§ 1355 Abs. 2 S. 2 BGB a.F. [1976]) - eine Regelung, die das Bundesverfassungsgericht vor dem Hintergrund des Gleichheitssatzes nicht eher als im Jahr 1991 für verfassungswidrig erklärte. ${ }^{60}$ Seit der vom Bundesverfassungsgericht eingeführten Möglichkeit einer getrennten Namensführung - die mit dem Gesetz zur Neuordnung des Familiennamensrechts ${ }^{61}$ auch in den Wortlaut des Bürgerlichen Gesetzbuchs überführt wurde, vgl. § 1355 Abs. 1 S. 3 BGB - ist die Ehefrau namensrechtlich nicht mehr zwangsweise Teil der Dynastie ihres Ehemanns. Dass der Gleichheitssatz eine namensrechtliche Gleichstellung der Ehegatten erfordert, hat im Übrigen auch der Europäische Gerichtshof für Menschenrechte bereits früh betont. ${ }^{62}$ Heute wäre ein im Mannesstamm dynastisches Ehenamensrecht bereits wegen der Öffnung der Ehe für gleichgeschlechtliche Paare ${ }^{63}$ ausgeschlossen.

Auch im Kindesnamensrecht war die dynastische Weitergabe des Namens im Mannesstamm vor dem Hintergrund des Gleichheitssatzes nicht mehr tragbar. Bereits nach dem Ersten Eherechtsreformgesetz konnte auch das eheliche Kind den Namen der Mutter als Geburtsnamen erhalten, wenn dieser auch Ehename war (vgl. § 1616 BGB a.F. [1976]). Seit 1998 und der großen Kindschaftsrechtsre-

56 Marianne Weber Ehefrau und Mutter in der Rechtsentwicklung - Eine Einführung (1907) S. 420.

57 Gesetz über die Gleichberechtigung von Mann und Frau auf dem Gebiet des bürgerlichen Rechts vom 18.6.1957, BGBl. 1957 I S. 609.

58 Erstes Gesetz zur Reform des Ehe- und Familienrechts vom 14.6.1976, BGBl. 1976 I S. 1421. 59 Siehe die Würdigung von Dieter Schwab 2017 - ein Jubiläumsjahr für das Familienrecht, FamRZ 2017, 1.

60 BVerfG 5.3.1991, BVerfGE 84, 9 = FamRZ 1991, 535.

61 Gesetz zur Neuordnung des Familiennamensrechts vom 16.12.1993, BGBl. 1993 I S. 2054.

62 EGMR 22.2.1994, Nr. 16213/90 (Case of Burghartz v. Switzerland); hierzu näher Walter Pintens Name und Menschenrechtskonvention, in: FS für Dieter Henrich (2000) S. 451 (454 f.).

63 Durch das Gesetz zur Einführung des Rechts auf Eheschließung für Personen gleichen Geschlechts vom 20.7.2017, BGBl. 2017 I S. 2787. 
form $^{64}$ koppelt das Gesetz den Geburtsnamen des Kindes an die Zuweisung der elterlichen Sorge. Der Name des Vaters und der Mutter stehen dabei grundsätzlich gleichberechtigt nebeneinander, wenn die Eltern keinen Ehenamen führen und ihnen die elterliche Sorge gemeinsam zusteht (vgl. §§ 1617, 1617b Abs. 1 BGB). Auch das Kind wurde damit namensrechtlich aus der Dynastie seines Vaters herausgelöst.

Infolge des verfassungsrechtlich verbürgten Gleichheitssatzes kann das Namensrecht damit keine Klassifizierungsfunktion im Hinblick auf die Zugehörigkeit des Namensträgers zu einer bestimmten Familiendynastie erfüllen; eine solche Zugehörigkeit wäre im Übrigen auch jenseits des Namensrechts juristisch irrelevant. Aber auch im Hinblick auf die Zugehörigkeit zu einer konkreten Kleinfamilie kann der Familienname keine verlässliche Auskunft mehr geben, da die verschiedenen Mitglieder der Kleinfamilie unterschiedliche Namen tragen können. Allenfalls noch bilateral zwischen einzelnen Familienmitgliedern kann der Familienname eine Verbindung dokumentieren. Aber auch das ist nicht zwingend der Fall. Wie bereits gesehen, müssen Ehegatten keinen Ehenamen führen. Auch Kinder können einen anderen Geburtsnamen tragen als ihre Eltern, etwa wenn der namensgebende Elternteil nach der Geburt einen vom anderen Ehegatten abgeleiteten Ehenamen erwirbt, ohne dass der Ehename auf den Geburtsnamen des Kindes nach $\S 1617$ c Abs. 1 BGB erstreckt oder das Kind nach § 1618 BGB einbenannt wird.

\section{b) Der Doppelname als Gebot der Gleichberechtigung von Mann und Frau}

Der Gleichheitssatz könnte aber noch weiter reichende Auswirkungen besitzen, gerade im Hinblick auf die Bildung des Namens in der Ehe und bei den gemeinsamen Kindern. Wie bereits gesehen, haben die Ehegatten nach $\S 1355$ Abs. 1 BGB die Wahl zwischen einer getrennten und gemeinsamen Namensführung. Entscheiden sich die Ehegatten für einen Ehenamen, dann kann der bei dieser Wahl „unterlegene“ Ehegatte - aber nur dieser - seinen Namen als Begleitnamen dem Ehenamen voranstellen oder anfügen (§ 1355 Abs. 4 S. 1 BGB). Umgangssprachlich spricht man vom Doppelnamen, was freilich juristisch unsauber ist, da es sich um eine Kombination von zwei rechtlich zu unterscheidenden Namenselementen handelt, dem Ehenamen und dem Begleitnamen. Nicht möglich ist ein echter Doppelname als gemeinsamer Ehename für beide Ehegatten, den diese auch an ihre Kinder weitergeben können. Auch einen Doppelnamen des Kindes

64 Gesetz zur Reform des Kindschaftsrechts vom 16.12.1997, BGBl. 1997 I S. 2942. 
bei getrennter Namensführung der Eltern - miteinander verheiratet oder nicht verbietet das Gesetz. Echte Doppelnamen, die auch weitergegeben werden können, kennt das deutsche Namensrecht nur selten, etwa, wie bereits gesehen (oben II.), bei der Einbenennung ( 1618 S. 2 BGB), aber auch bei der Adoption ( 1757 Abs. 3 S. 1 Nr. 2 BGB). Dennoch begegnet man bei einem Ritt durchs namensrechtliche Absurdistan in Deutschland auch den folgenden Konstellationen, in denen Doppelnamen erstaunlicherweise möglich sind: Ein echter Doppelname kann entstehen, wenn ein Ehegatte nach Beendigung der ersten Ehe weiterhin den ehemaligen Ehenamen mit Begleitnamen führt (nach $\S 1355$ Abs. 5 S. 1 oder S. 2 Fall 3 BGB) und die Ehegatten diesen „geführten Namen“ bei einer erneuten Eheschließung zum Ehenamen bestimmen (§1355 Abs. 2 Fall 2 BGB). ${ }^{65}$ Diesen Doppelnamen geben die Ehegatten dann auch zwingend an ihre gemeinsamen Kinder als Geburtsnamen weiter. ${ }^{66}$ Ebenso kreiert das deutsche Namensrecht einen echten Doppelnamen als Geburtsnamen eines Kindes, wenn die Eltern keinen Ehenamen führen, aber der namensgebende Elternteil einen Ehenamen mit Begleitnamen führt und (bei gemeinsamer Sorge der Eltern) die Eltern dem Kind diesen geführten Namen gemäß $§ 1617$ Abs. 1 S. 1 BGB erteilen oder (bei Alleinsorge des namensgebenden Elternteils) das Kind diesen Namen von Gesetzes wegen gemäß § 1617a Abs. 1 BGB erhält. Ein - namensrechtsspezifischer - sachlicher Grund, warum gerade in diesen Ausnahmefällen die Erwägungen gegen Doppelnamen zurücktreten sollen, nicht aber bei aus den Eltern- oder Ehegattennamen zusammengesetzten Doppelnamen, ist nicht ersichtlich. ${ }^{67}$

Nun kann man sich über Doppelnamen herrlich amüsieren, vor allem weil sich recht einfach lächerliche Namenskombinationen bilden lassen. ${ }^{68}$ Eine sehr lesenswerte (zeit)historische Skizze von Martin Otto - Titel: „Wer hat Angst vor Müller-Lüdenscheidt?“ - hat kürzlich gezeigt, dass es bisher vor allem diese Ridikülisierungsneigung einer jeden Doppelnamensdiskussion (gepaart mit einer Skepsis gegen „Bindestrich-Ehefrauen“) war, die den Gesetzgeber von der Ein-

65 Näher Hepting/Dutta (Fn. 6) Rn. III-583.

66 Näher Hepting/Dutta (Fn. 6) Rn. III-583.

67 Anders BVerfG 30.1.2002, BVerfGE 104, 373 = StAZ 2002, 72 = FamRZ 2002, 306 (311); unverständlich ist meines Erachtens insbesondere, warum das Bundesverfassungsgericht meint, dass bei der Einbenennung die Verbindung zum namensgebenden Elternteil und zum Stiefelternteil sich von der Verbindung zu beiden Elternteilen unterscheidet und so stark ist, dass sie einen Doppelnamen rechtfertigt, vgl. auch Hilbig-Lugani (Fn. 20) § 1617 BGB Rn. 25; skeptischer offenbar auch BVerfG 5.5.2009, BVerfGE 123, 90 = StAZ 2009, 179 = FamRZ 2009, 939 (942).

68 Geradezu legendär ist der Text von Dieter Schwab Statt einer Glosse: Der Name ist Schall und Rauch, FamRZ 1992, 1015; zur Wirkgeschichte der Schwabschen Glosse gerade auch auf das konkret aufgespießte Gesetzgebungsverfahren siehe Thomas Wagenitz Über Schall, Rauch und andere Unwägbarkeiten, in: FS für Dieter Schwab (2005) S. 443. 
führung des Doppelnamens abgehalten hat. ${ }^{69}$ Diese deutsche Doppelnamensaversion überrascht. In anderen Rechtsordnungen stellt der Doppelname das Grundmodell dar, wenn man nur in die spanischsprachige Welt blickt, ${ }^{70}$ oder ist ohne Weiteres zulässig, wie etwa seit einigen Jahren in Österreich. ${ }^{71}$ Eine überwältigende Mehrheit der Mitgliedstaaten der Europäischen Union erlaubt einen Doppelnamen als Geburtsnamen eines Kindes. ${ }^{72}$ Dabei ist, und das berichtet auch die Personenstandspraxis, der Wunsch nach einem Doppelnamen in Deutschland groß, vor allem für die gemeinsamen Kinder, zu denen oftmals beide Elternteile ein namensrechtliches Band besitzen wollen - ein Gedanke, der auch zu einem partnerschaftlichen Elternbild passt. ${ }^{73}$

Es spricht sogar Einiges dafür, dass das Verbot des Doppelnamens in der Paar- und Elternbeziehung zur Perpetuierung letzter patriarchalischer Strukturen beiträgt: Untersuchungen legen den Schluss nahe, dass Ehefrauen und Mütter sei es bei der Bestimmung des Ehenamens (und damit auch beim Geburtsnamen der gemeinsamen Kinder) oder isoliert bei der Bestimmung des Geburtsnamens der Kinder - namensrechtlich zurückstecken und sich auch heute noch regelmäßig der Mannes- oder Vatername durchsetzt. So diagnostiziert etwa Saskia Lettmaier in einem kürzlich erschienenen Aufsatz in Deutschland ,patronymische Namenskonventionen“774. Sie kommt nach einer nicht repräsentativen Um-

69 Martin Otto Wer hat Angst vor Müller-Lüdenscheidt? - Vom (bisherigen) Scheitern einer Legalisierung „echter Doppelnamen“ in Deutschland, StAZ 2019, 257.

70 Siehe für Spanien bei einem Kind mit feststehender Mutter- und Vaterschaft die Regelung in Art. 49 des Ley del registro civil.

$71 \S 93$ Abs. 2 des Allgemeinen Bürgerlichen Gesetzbuchs.

72 Siehe - neben den Nachweisen in den beiden vorigen Fn. - etwa Art. $335 \S 1$ Abs. 1 des belgischen Code civil; § 8 des dänischen Navneloven; $§ 5$ des finnischen Etu- ja sukunimilaki; Art. 311-21 Abs. 1 des französischen Code civil; Art. 1505 Abs. 2 des griechischen Astikos kōdikas; first schedule zum irischen Civil Registration Act 2004; Art. 3 Abs. 1 S. 2 des kroatischen Zakon o osobnom imenu; Art. 57 Abs. 3 S. 2 des luxemburgischen Code civil; Art. 4 Abs. 13 lit. b, Abs. 14 des maltesischen Civil code; Art. 88 § 1 S. 3 des polnischen Kodeks rodzinny i opiekuńczy; Art. 1875 des portugiesischen Código civil; Art. 449 Abs. 2 S. 1 des rumänischen Codul civil; § 4 Abs. 1 Nr. 3 des schwedischen Lag om personnamn; Art. 7 Abs. 3 des slowenischen Zakon o osebnem imenu; § 4:150 Abs. 1 S. 3 und 4 des ungarischen Polgári Törvénykönyv.

73 von Sachsen Gessaphe (Fn. 21) § 1617 BGB Rn. 43; vgl. etwa bereits Dieter Giesen Der Familienname aus rechtshistorischer, rechtsvergleichender und rechtspolitischer Sicht, FuR 1993, 65 (78 f.). Auch BVerfG 30.1.2002, BVerfGE 104, 373 = StAZ 2002, 72 = FamRZ 2002, 306 (309) betont: „Der aus den Namen der Eltern zusammengesetzte Doppelname vermag sogar noch besser als ein aus den beiden Namen gewählter Geburtsname die familiäre Zugehörigkeit des Kindes auszudrücken, dokumentiert er doch die Verbundenheit des Kindes mit beiden Elternteilen im Namen“. 74 Saskia Lettmaier Notwendigkeit einer Reform des (Familien-)Namensrechts? FamRZ 2020, 1 (7). 
frage bei dreizehn deutschen Standesämtern zu dem Ergebnis, dass sich etwa 80 Prozent der Ehepaare für einen Ehenamen entscheiden, wobei 92 Prozent der verschiedengeschlechtlichen Paare den Mannesnamen als Ehenamen wählen. Auch das Bundesverfassungsgericht qualifizierte bereits im Jahr 2002 den Umstand, „daß Ehegatten sich immer noch bei der Wahl des Geburtsnamens ihres Kindes überwiegend für den Namen des Mannes entscheiden, möglicherweise [als] Ausdruck eines tradierten Rollenverständnisses“, das „darauf hindeute [...], daß insoweit bei der von Art. 6 I GG geschützten freien und selbstverantwortlichen Entscheidung der Ehegatten über die Ausgestaltung ihres Verhältnisses zueinander und zum Kind sowie über ihre Aufgabenverteilung in der Ehe [...] faktisch noch keine gleichberechtigte Partnerschaft besteht. "75 Die Hoffnung, dass seit der Abschaffung des Zwangs zum gemeinsamen Ehenamen (soeben IV. 2. a) Frauen „ihrem gewandelten Selbstverständnis entsprechend in der Namensführung auch faktisch gleichberechtigt" sind, ${ }^{76}$ hat sich offenbar nicht erfüllt. Manifestiert sich hier eine strukturelle Unterlegenheit der Ehefrau und Mutter, die - um Konflikte $\mathrm{zu}$ vermeiden und sozialen Konventionen zu genügen - auf ihren Namen verzichtet?

Würde diese naheliegende Annahme zutreffen, dann müsste auch aus Gleichbehandlungserwägungen über eine Reform des derzeitigen Namensrechts nachgedacht werden. ${ }^{77}$ Dabei ginge es aber zu weit, wie von den Grünen im Jahr 1989 vorgeschlagen, gemeinsame Namen in der Paar- und Elternbeziehung zu verbieten, etwa durch eine zwingend getrennte Namensführung von Ehegatten (,Jede Person behält grundsätzlich - auch bei Eheschließung - ihren Geburtsnamen“) und die zwingende Weitergabe des Namens im Frauenstamm (,Kinder erhalten den Namen der Mutter“), um „patriarchale“ Strukturen im Namensrecht aufzubrechen und Frauen nicht mehr - „oft nur um des ,Familienfriedens willen““ - zur Aufgabe ihres Namens zu drängen. ${ }^{78}$ Dieser Vorschlag dürfte auch verfassungsrechtlich unzulässig sein. ${ }^{79}$ Ein milderes Mittel wäre die Zulässigkeit von

75 BVerfG 30.1.2002, BVerfGE 104, 373 = StAZ 2002, 72 = FamRZ 2002, 306 (310).

76 Nina Dethloff/Susanne Walter Abschied vom Zwang zum gemeinsamen Ehenamen, NJW 1991, 1575 (1579).

77 Vgl. bereits zur mittelbaren Benachteiligung der Frau im Namensrecht Ute Sacksofsky Das Ehenamensrecht zwischen Tradition und Gleichberechtigung - zum neuen Ehenamensurteil des BVerfG, FPR 2004, 371 (374).

78 Antrag der Fraktion der Grünen zum Namensrecht vom 26.4.1989, BT-Drucks. 11/4437; in eine ähnliche Richtung (,unter sachlich-pragmatischem Aspekt erweist sich jedoch die Anknüpfung an den Mutternamen als überlegen“) zuvor bereits Coester (Fn. 43) 304, allerdings mit der damals noch durchaus zutreffenden Beobachtung, dass nach dem Scheitern der Elternbeziehung regelmäßig die Mutter die zentrale Zuordnungsperson für das Kind ist.

79 Gaaz (Fn. 18) 165 in Fn. 63. 
Doppelnamen, auch als Gebot der Gleichberechtigung von Mann und Frau, wie es in Art. 3 Abs. 2 GG niedergelegt ist. ${ }^{80}$ Zwar hat das Bundesverfassungsgericht im Jahr 2002 das Doppelnamensverbot beim Ehe- und Geburtsnamen noch gehalten, ${ }^{81}$ ähnlich wie der Europäische Gerichtshof für Menschenrechte. ${ }^{82}$ Aber jedenfalls das Bundesverfassungsgericht hat mittlerweile für mittelbare Diskriminierungen im Personenstandsrecht ein sehr feines Gespür. Losgelöst von der Frage, ob der „Doppelname als Menschenrecht“83 anzusehen ist, lässt sich das Verbot der Doppelnamen zumindest rechtspolitisch nicht mehr halten, ${ }^{84}$ wie nun auch das Eckpunktepapier des Bundesinnenministeriums und des Bundesjustizministeriums betont; ${ }^{85}$ seit April 2020 liegt sogar ein Gesetzesentwurf der Freien Demokraten vor, der echte Doppelnamen für Ehepaare und Kinder einführen möchte. ${ }^{86}$

80 Siehe bereits Hildegard Krüger Der Name der Frau nach bürgerlichem Recht, AcP 156 (1957) 232 (257); Sacksofsky (Fn. 77) 375; dies. Das Bundesverfassungsgericht und das Familiennamensrecht - Ein Schritt zurück im Zickzackkurs, FPR 2010, 15 (19 f.); so auch Lettmaier (Fn. 74) 8 ff.; auch in der Begründung des Entwurfs eines Gesetzes zur Neuordnung des Familiennamensrechts (Fn. 48) S. 12, klingt dieser Gedanke an: „Die Möglichkeit eines solchen Doppelnamens gestattet den Ehegatten Kompromisse bei der Bestimmung des künftigen gemeinsamen Familiennamens: Der Doppelname spiegelt nicht nur die mit der Eheschließung bewirkte gleichberechtigte Einheit der Ehegatten; er vermittelt jedem der Ehegatten auch subjektiv das Gefühl, sich in seinem neuen nunmehr gemeinsamen - Familiennamen ,wiederzufinden““.

81 BVerfG 30.1.2002, BVerfGE 104, 373 = StAZ 2002, 72 = FamRZ 2002, 306 (308 ff.); nochmals bestätigt in BVerfG 5.5.2009, BVerfGE 123, 90 = StAZ 2009, 179 = FamRZ 2009, 939 (941).

82 EGMR 6.5.2008, Nr. 33572/02 (Alexandra von Rehlingen and others against Germany), deutsche Übersetzung abgedruckt in StAZ 2008, 375; zu dieser Entscheidung Berthold Gaaz Zum Recht des Kindesnamens in Europa, StAZ 2008, 365.

83 Berthold Gaaz Der Doppelname als Menschenrecht? - Zum Recht des Kindesnamens in Europa, in: FS für Rainer Frank (2008) S. 381.

84 Für die Zulässigkeit von Doppelnamen etwa Nina Dethloff/Susanne Walther Der Ehename im Lichte von Persönlichkeitsrecht und Gleichberechtigung, EuGRZ 1987, 41 (47); Günther Grasmann Zur Reform des Ehe- und Familiennamens im deutschen Sach- und Verweisungsrecht, ZRP 1990, 12 (14 f.); Schwenzer (Fn. 43) 395, 396; Dagmar Coester-Waltjen „Auf dass Ihr euch ewig bindet...“Das Ehenamensrecht, Jura 2007, 586 (589); siehe auch die differenzierten Vorschläge für Begleitnamen von Robert Battes Der Weg aus der Sackgasse - Vorschlag für eine gründliche Reform des deutschen Namensrechts, FamRZ 2008, 1037; ders. Prolegomena zu einer grundlegenden Reform des deutschen Namensrechts, in: FS für Harm Peter Westermann (2008) S. 93 (107 f.); Hilbig-Lugani (Fn. 20) § 1617 BGB Rn. 25; von Sachsen Gessaphe (Fn. 22) § 1355 BGB Rn. 72; ders. (Fn. 21) § 1617 BGB Rn. 43 f.

85 Eckpunkte zur Reform des Namensrechts (Fn. 2) S. 7 unter III. 6.

86 Siehe den Entwurf eines Gesetzes zur Änderung des Ehe- und Geburtsnamensrechts - Echte Doppelnamen für Ehepaare und Kinder, BT-Drucks. 19/18314. 


\section{Elternrecht und allgemeines Persönlichkeitsrecht}

Einer Klassifizierungsfunktion des Namens werden auch durch andere höherrangige Prinzipien Grenzen gesetzt. Das betrifft etwa den Vornamen des Kindes, der, wie bereits erwähnt, traditionell auch über das Geschlecht des Kindes Auskunft geben sollte (oben III. 1.). Allerdings kam das Bundesverfassungsgericht zu dem Ergebnis, dass diese Klassifizierungsfunktion mit den in Art. 6 Abs. 2 GG verbürgten Elternrechten unvereinbar ist. Grenze bei der Vornamenswahl kann allein das Kindeswohl sein, ${ }^{87}$ sodass vor allem ein geschlechtsneutraler Vorname zulässig ist, anders als womöglich ein geschlechtswidriger.

Auch die zunehmende Bedeutung des „Bands zwischen Person und Name“ 88 reflektiert das Verfassungsrecht: Der Schutz unserer Persönlichkeit umfasst auch den Namen, sei es im deutschen Verfassungsrecht als Element des allgemeinen Persönlichkeitsrechts nach Art. 2 Abs. 1 GG in Verbindung mit Art. 1 Abs. 1 GG $^{89}$ oder in der Europäischen Menschenrechtskonvention ${ }^{90}$ (EMRK) und in der europäischen Grundrechtecharta ${ }^{91}$ (GRCh) als Teil des Privat- und Familienlebens nach Art. $8 \mathrm{EMRK}^{92}$ und Art. $7 \mathrm{GRCh}^{93}$. Wenig überraschend beschränkt deshalb auch das allgemeine Persönlichkeitsrecht des Namensträgers eine Klassifizie-

87 Vor allem BVerfG 3.11.2005, StAZ 2006, 50 = FamRZ 2005, 2049 und BVerfG 5.12. 2008, StAZ 2009, 76 = FamRZ 2009, 294; so auch bereits BVerfG 30.1.2002, BVerfGE 104, 373 = StAZ 2002, $72=$ FamRZ 2002, 306 (308).

88 Vgl. auch Walter Pintens Namensrecht, in: Jürgen Basedow/Klaus Hopt/Reinhard Zimmermann (Hrsg.) Handwörterbuch des Europäischen Privatrechts, Band II (2009) S. 1093 (1094) (,das Band zwischen der Person und ihrem Namen“ wird „stärker akzentuiert“).

89 Etwa BVerfG 30.1.2002, BVerfGE 104, 373 = StAZ 2002, 72 = FamRZ 2002, 306 (308); BVerfG 18.2.2004, BVerfGE 109, 256 = StAZ 2004, 104 = FamRZ 2004, 515 (516); BVerfG 5. 5. 2009, BVerfGE 123, 90 = StAZ 2009, 179 = FamRZ 2009, 939 (940); so bereits Krüger (Fn. 80) 242 („Das ist so allgemein anerkannt, daß sich Zitate erübrigen“).

90 Konvention zum Schutze der Menschenrechte und Grundfreiheiten vom 4.11.1950, BGBl. 1952 II S. 685.

91 Charta der Grundrechte der Europäischen Union vom 12.12.2007, ABl. 2007 C 303/1.

92 EGMR 22.2.1994, Nr. 16213/90 (Case of Burghartz v. Switzerland) Rn. 24; EGMR 25.11.1994, Nr. 18131/91 (Case of Stjerna v. Finland) Rn. 37.

93 EuGH 22.12. 2010, Rs. C-208/09 (Ilonka Sayn-Wittgenstein gegen Landeshauptmann von Wien) Slg. 2010, I-13693 = StAZ 2011, 77 = FamRZ 2011, 1486, Rn. 52; EuGH 12.5.2011, Rs. C-391/09 (Malgožata Runevič-Vardyn, Łukasz Paweł Wardyn gegen Vilniaus miesto savivaldybės administracija, Lietuvos Respublikos teisingumo ministerija, Valstybinė lietuvių kalbos komisija, Vilniaus miesto savivaldybės administracijos Teisès departamento Civilinės metrikacijos skyrius) Slg. 2011, I-3787 = StAZ 2011, 274 = FamRZ 2011, 1038, Rn. 66; EuGH 2.6. 2016, Rs. C-438/14 (Nabiel Peter Bogendorff von Wolffersdorff gegen Standesamt der Stadt Karlsruhe und Zentraler Juristischer Dienst der Stadt Karlsruhe) StAZ 2016, 203 = FamRZ 2016, 1239, Rn. 35. 
rungsfunktion des Familiennamens im Hinblick auf die Familienzugehörigkeit. Das Bundesverfassungsgericht betonte im Jahr 2004, dass bei der Wahl des Ehenamens die Ehegatten nicht nur auf ihre Geburtsnamen beschränkt sein dürfen, sondern auch den so genannten Präsenznamen wählen können müssen, also den Familiennamen, den ein Ehegatte zum Zeitpunkt der Eheschließung führt, ${ }^{94}$ beispielsweise den durch eine Vorehe erworbenen Ehenamen, der nach Beendigung der Vorehe von dem Ehegatten weitergeführt wurde ( $§ 1355$ Abs. 5 S. 1 BGB), gegebenenfalls sogar in Verbindung mit einem Begleitnamen (siehe oben IV. 2. b). Der Gesetzgeber reagierte mit einer entsprechenden Neufassung des $\S 1355$ Abs. 2 BGB. ${ }^{95}$ Sogar Ehenamen aus weiter zurückliegenden Ehen können nunmehr als Ehenamen an einen neuen Ehegatten weitergegeben werden. ${ }^{96}$

\section{Integration}

Auch die Individualisierungsfunktion des Namens steht im geltenden Namensrecht nicht mehr uneingeschränkt zur Verfügung, auch jenseits des Konflikts mit der Klassifizierungsfunktion (oben III. 1.). Bereits die Möglichkeit einer isolierten Namensänderung aus wichtigem Grund nach dem Namensänderungsgesetz (oben II.) weist darauf hin, dass die Namenskontinuität auch namensrechtsfremden Belangen weichen muss, beispielsweise dem allgemeinen Persönlichkeitsrecht des Namensträgers, das durch ein Festhalten an einem unzumutbaren Namen beeinträchtigt wird. ${ }^{97}$

Darüber hinaus hat der Gesetzgeber die Namenskontinuität erheblich relativiert, etwa um eine namensrechtliche Integration von Neubürgern zu fördern. Das deutsche Namenskollisionsrecht folgt dem Staatsangehörigkeitsprinzip. Nach Art. 10 Abs. 1 EGBGB unterliegt der Name der Person dem Recht des Staates, dem der Namensträger angehört. Erwirbt eine Person nachträglich die deutsche Staatsangehörigkeit, so wechselt das anwendbare Namensrecht; der nach ausländischem Recht erworbene Name unterliegt fortan deutschem Recht. Allerdings

94 BVerfG 18.2.2004, BVerfGE 109, 256 = StAZ 2004, 104 = FamRZ 2004, 515 m. kritischer Anm. Jan von Hein (,Das vorliegende Urteil des BVerfG ist einem Maße verfehlt, dass es zunächst schwerfällt, sich ihm anders als satirisch zu nähern“).

95 Durch das Gesetz zur Änderung des Ehe- und Lebenspartnerschaftsnamensrechts vom 6.2. 2005, BGBl. 2005 I S. 203.

96 Näheres etwa bei Hepting/Dutta (oben Fn. 6) Rn. III-580.

97 Siehe auch die Fallgruppen für einen wichtigen Grund für eine Namensänderung in Ziff. $33 \mathrm{ff}$. der Allgemeinen Verwaltungsvorschrift zum Gesetz über die Änderung von Familiennamen und Vornamen (oben Fn. 29). 
sieht seit 2007 und der großen Personenstandsrechtsreform ${ }^{98}$ Art. 47 EGBGB eine Angleichung des Namens an die inländische Namenstypologie vor. Etwa kann der Neubürger nach Art. 47 Abs. 1 S. 1 Nr. 4 und Nr. 5 EGBGB die ursprüngliche Form eines nach dem Geschlecht oder dem Verwandtschaftsverhältnis abgewandelten Namens annehmen („Tschaikowski“ statt „Tschaikowskaja“) und sogar für eine deutschsprachige Form seines Vor- oder Familiennamens optieren („Peter Schmidt“ statt „Pjotr Šmit“). Der Statutenwechsel ermöglicht mithin in Grenzen eine isolierte Namensänderung.

\section{Personenfreizügigkeit und Diskriminierungsverbot}

Eine erhebliche Einschränkung hat sowohl die Individualisierungs- als auch die Klassifizierungsfunktion des Namens durch das Unionsrecht erfahren. In mehreren Entscheidungen hat der Gerichtshof der Europäischen Union ${ }^{99}$ klargestellt, dass die Unterschiede in den Namensrechten der Mitgliedstaaten die Personenfreizügigkeit (Art. 21 Abs. 1 AEUV) beschränken und gegen das Diskriminierungsverbot (Art. 18 AEUV) verstoßen können, wenn Unterschiede vor allem auch der mitgliedstaatlichen Namenskollisionsrechte zu hinkenden Namensverhältnissen führen und ein und derselbe Unionsbürger in unterschiedlichen Mitgliedstaaten unterschiedliche Namen trägt. ${ }^{100}$ Kurzgefasst folgert der Gerichtshof, dass ein in einem Mitgliedstaat tatsächlich erlangter Name in den anderen Mitgliedstaaten anzuerkennen ist. ${ }^{101}$

Mittlerweile hat der deutsche Gesetzgeber versucht, dieses Anerkennungsgebot in einem neuen Art. 48 EGBGB umzusetzen, der es einem Namensträger unter bestimmten Voraussetzungen gestattet, einen in einem anderen Mitglied-

98 Gesetz zur Reform des Personenstandsrechts vom 19.2.2007, BGBl. 2007 I S. 122.

99 Vor allem EuGH 2.10.2003, Rs. C-148/02 (Carlos Garcia Avello gegen Belgischer Staat) Slg. 2003, I-11613 = StAZ 2004, 40 = FamRZ 2004, 173; EuGH 14.10.2008, Rs. C-353/06 (Stefan Grunkin und Dorothee Regina Paul) Slg. 2008, I-7639 = StAZ 2009, 9 = FamRZ 2008, 2089; EuGH 22.12. 2010, Rs. C-208/09 (Ilonka Sayn-Wittgenstein gegen Landeshauptmann von Wien) Slg. 2010, $\mathrm{I}-13693$ = StAZ 2011, 77 = FamRZ 2011, 1486; EuGH 2.6.2016, Rs. C-438/14 (Nabiel Peter Bogendorff von Wolffersdorff gegen Standesamt der Stadt Karlsruhe und Zentraler Juristischer Dienst der Stadt Karlsruhe) StAZ 2016, 203 = FamRZ 2016, 1239.

100 Hierauf hat übrigens bereits Dietrich Nelle Der Familienname - Perspektiven für eine rechtsvereinheitlichende Reform (Teil II), FamRZ 1990, 935 (940) hingewiesen.

101 So bereits Dieter Henrich Das internationale Namensrecht auf dem Prüfstand des EuGH, FS für Andreas Heldrich (2005) S. 667 (675); ein Überblick über die bisherige namensrechtliche EuGH-Rechtsprechung findet sich bei Anatol Dutta Namenstourismus in Europa? FamRZ 2016, 1213 (1214 ff.). 
staat erworbenen und dort in einem Personenstandsregister eingetragenen Namen zu wählen. Diese Vorschrift setzt das unionsrechtliche Anerkennungsgebot nur unzureichend um. ${ }^{102}$ Sinnvoller wäre es, der Unionsgesetzgeber würde reagieren und durch eine Vereinheitlichung des Namenskollisionsrechts und die Einführung eines Anerkennungsprinzips das Problem an der Wurzel packen. ${ }^{103}$ In anderen Mitgliedstaaten spielen die Individualisierungs- und Klassifizierungsfunktion des Namens eine geringere Rolle als bei uns, bis zum vollständigen Vollzug des Austritts des Vereinigten Königreichs aus der Europäischen Union ${ }^{104}$ etwa noch in England und Wales, dessen Namensrecht dem Einzelnen über das deed-poll-Verfahren grundsätzlich eine freie Wahl seines Namens gestattet. ${ }^{105}$ Das Unionsrecht ermöglicht damit einen gewissen Namenstourismus, zumal der Gerichtshof für das deutsche Namensrecht betont hat, dass die Namenskontinuität (Identifikationsfunktion, oben III. 1.) und die Offenlegung von Familienbeziehungen (Klassifizierungsfunktion, oben III. 1.), aber auch die Begrenzung mehrgliedriger Namen (Ordnungsinteresse des Staates, oben III. 2.) keine Gründe sind, die einen Eingriff in die Personenfreizügigkeit und einen Verstoß gegen das Diskriminierungsverbot rechtfertigen. ${ }^{106}$

102 Siehe für eine Fallgruppe EuGH 8.6.2017, Rs. C-541/15 (Mircea Florian Freitag) StAZ 2017, 338 = FamRZ 2017, 1175; daneben ist Art. 48 EGBGB auch in weiterer Hinsicht zu eng gefasst, um die Rechtsprechung des Gerichtshofs im deutschen Recht vollständig zu implementieren, im Einzelnen Hepting/Dutta (Fn. 6) Rn. II-451 ff.

103 Ein Vorschlag für eine europäische Verordnung zum internationalen Namensrecht findet sich bei Anatol Dutta/Rainer Frank/Robert Freitag/Tobias Helms/Karl Krömer/Walter Pintens Ein Name in ganz Europa - Entwurf einer Europäischen Verordnung über das Internationale Namensrecht, StAZ 2014, 33; englische Kurzfassung (One Name Throughout Europe - Draft for a European Regulation on the Law Applicable to Names) in YPIL 15 (2013/2014) 31 sowie französische Kurzfassung (Un nom dans toute l'Europe - Une proposition de règlement européen sur le droit international du nom) in Rev. crit. dr. int. pr. 2014, 733; zu dem Vorschlag siehe auch Anatol Dutta/Tobias Helms/Walter Pintens (Hrsg.) Ein Name in ganz Europa - Vorschläge für ein Internationales Namensrecht der Europäischen Union (2016).

104 Zur Fortwirkung der Problematik womöglich auch nach Ablauf des Übergangszeitraums Anatol Dutta Brexit und Standesamt: Bye-bye? Vorerst nicht, StAZ 2020, 65 (66 f.).

105 Details bei Saskia Lettmaier Personennamen und Recht in Großbritannien aus rechtswissenschaftlicher Sicht, StAZ 2015, 289.

106 Siehe jüngst vor allem den EuGH 2.6.2016, Rs. C-438/14 (Nabiel Peter Bogendorff von Wolffersdorff gegen Standesamt der Stadt Karlsruhe und Zentraler Juristischer Dienst der Stadt Karlsruhe) StAZ 2016, 203 = FamRZ 2016, 1239, Rn. 48 ff. 